\section{(2) OPEN ACCESS}

\title{
Metabolomic profiling identifies plasma sphingosine 1-phosphate levels associated with welding exposures
}

\author{
Shangzhi Gao (1) , ${ }^{1}$ Zhu Zhuo, ${ }^{2}$ John Hutchinson, ${ }^{2}$ Li Su, ${ }^{1}$ David C Christiani (1) 1,3
}

- Additional material is published online only. To view, please visit the journal online (http://dx.doi.org/10.1136/ oemed-2020-106918).

${ }^{1}$ Environmental Health, Harvard University T H Chan School of Public Health, Boston, Massachusetts, USA

${ }^{2}$ Biostatistics, Harvard University T H Chan School of Public Health, Boston, Massachusetts, USA

${ }^{3}$ Pulmonary and Critical Care Division, Department of Medicine, Massachusetts General Hospital, Boston, Massachusetts, United States

\section{Correspondence to} Dr David C Christiani Environmental Health, Harvard University T H Chan School of Public Health, Boston, Massachusetts 02115, USA; dchris@hsph.harvard.edu

Received 26 July 2020 Revised 22 September 2020 Accepted 2 October 2020 Published Online First 26 October 2020

\author{
ABSTRACT \\ Background Despite a number of known health \\ hazards of welding fume exposure, it is unclear how \\ exposure affects the human metabolome. \\ Objective We assessed the metabolic profiles of \\ welders before and after a 6-hour welding shift, \\ controlling for circadian rhythm of metabolism on a non- \\ welding day.
}

Methods Welders were recruited from a training centre in Quincy, Massachusetts, in 2006 and 2010-2012 and donated blood samples on a welding shift day before and after work, as well as on a non-welding day spent in an adjacent classroom. In total, we collected 509 samples from 74 participants. Liquid chromatographymass spectrometry quantified 665 metabolites from thawed plasmas. Metabolites with significant time (afternoon compared with morning) and day (welding/ classroom) interactions were identified by two-way analysis of variance, and the overnight changes were evaluated.

Results Sphingosine 1-phosphate (S1P) and sphingasine 1-phosphate (SA1P) exhibited significant interaction effects between day and time with false discovery rate-adjusted $p$ values of 0.03 and $<0.01$, respectively. S1P, SA1P and sphingosine shared similar trends over time: high relative levels in the morning of a non-welding day declining by afternoon, but with lower starting levels on a welding day and no decline. There was no obvious pattern related to current smoking status.

Conclusion S1P and SA1P profiles were different between welding day and classroom day. The S1P pathway was disrupted on the day of welding exposure. The levels of S1P, SA1P and sphingosine were highly correlated over time. S1P is a signalling lipid with many vital roles; thus, the underlying mechanism and clinical implications of this alteration need further investigation.

\section{INTRODUCTION}

Welding is a fabrication or sculptural process that joins metal materials by melting parts with high heat followed by fusion after cooling. Heating metals above their melting points generate potentially hazardous vapours and fine particle condensates, and the occupational hazards of welding includes such toxic fumes and gases as well as ultraviolet radiation, electromagnetic fields and coexposure to asbestos and harmful solvents. The type and extent of exposure relate to the process performed, material used, ventilation and enclosure of the space,

\section{Key messages}

What is already known about this subject?

- Welding fume has been classified as a group 1 carcinogen by the International Agency for Research on Cancer. Preliminary studies suggest that it is also associated with occupational respiratory diseases, systemic inflammation, DNA damage, DNA methylation and disrupted heart rate variability.

What are the new findings?

- This repeated measurement metabolomic profiling study showed that sphingosine 1-phosphate (S1P) and sphingasine 1-phosphate profiles were different between welding days and days without direct welding fume exposure. Welding fume may disrupt the circadian rhythm of metabolites in the S1P pathway.

How might this impact on policy or clinical practice in the foreseeable future?

- Foreseeable clinical/public health impact is to advise reductions in exposure below those that are required by current regulation.

and use of personal protective equipment. Welding puts approximately 11 million welders worldwide at risk and exposes around 110 million additional workers to welding-related toxins. ${ }^{1}$

Welding exposure has many known adverse health effects. Welding fumes and ultraviolet radiation have been classified as group 1 carcinogens by the International Agency for Research on Cancer ${ }^{1}$ and are associated with increased risks of ocular cancer, lung cancer and liver cancer. Welding fumes also cause occupational respiratory diseases. Rhinitis and laryngitis can develop from deposition of large particles in the upper respiratory tract and absorption of soluble gases, including sodium dioxide by mucus membranes of the upper respiratory tract ${ }^{2}$; bronchitis can develop when particulates of $\leq 2.5 \mu \mathrm{m}$ are deposited in bronchioles and parenchyma. Epidemiological studies have also shown associations between welding fumes and systemic inflammation, ${ }^{34}$ DNA damage biomarkers, ${ }^{56}$ heart rate variability ${ }^{7-9}$ and epigenetic effects. ${ }^{10-12}$

There are exposure limits for specific materials used in welding but no Occupational Safety and 
Health Administration (OSHA) occupational exposure limit for generic welding fume exposure due to variation in welding materials and methods. There is also a knowledge gap with regard to the dose-response relationship of welding fumes on disease risk, and there is limited information about how welding fume exposure may affect the human metabolome.

Metabolomics is a powerful analytical approach to reveal physiological and pathological metabolic alterations. It can be used to identify disease biomarkers and pathophysiological mechanisms using biological samples. Metabolomics can be a rigorous tool to study the toxicology of welding fume exposure and the mechanism of disease development. However, to achieve rigour, biological variation from interpersonal and intrapersonal (eg, circadian rhythm) sources must be sufficiently controlled. This represents the major challenge affecting studies linking metabolic alterations to environmental exposures. In this study, we examine the metabolic profile associated with welding fume exposure controlled via comparison to a time-matched point on a day without exposure. Also, we assessed the profile of differentially expressed metabolites during exposure, as well as overnight profiles before and after the exposure.

\section{METHODS}

\section{Study population and study design}

We invited apprentices and instructors attending a welding skill training centre to participate in our study. The training centre was owned and operated by the International Brotherhood of Boilermakers Union, Local 29, in Quincy, Massachusetts, and our participant pool consisted of active and inactive members of the Union. They performed welding, cutting and repairing jobs in commercial power plants for a living.

Data were collected in 2006 and from 2010 to 2012. Samples were collected periodically during ongoing training programmes. The regular maintenance routine of power plants was in spring and autumn, so the training programme was usually scheduled on the weekends of off seasons, namely, summer and winter. The study design and sample collection are shown in table 1. Briefly, in 2006, on the day of a shift, we collected blood samples at 06:30-7:00 before a 6-hour shift and at 142:30-15:00 after work and then again at 6:30-7:00 of the following day. In 2010, we collected samples in the afternoon of the day prior to the shift and then again before and after work on the day of the shift. During 2011 and during 2012, on the day of a shift, we collected samples before and after work to be compared with control samples taken within 1 day before or 6 days after the welding day and from a matched time period spent in an adjacent classroom area separated from the work area by a wall and with separate exhaust ventilation. If control and exposure days were adjacent, data were also qualified for use in evaluating an overnight metabolic profile. A few participants also provided additional baseline samples that were measured in the afternoon of non-welding days. There were a total of 509 qualified samples collected from 74 participants, some of whom joined in multiple study periods. Among these samples, $176(44 \times 4)$ samples were qualified for two-factor analysis of variance (ANOVA), $297(99 \times 3)$ samples were qualified for pre-exposure overnight profile, and $132(44 \times 3)$ samples were qualified for postexposure overnight profile (table 1). Some participants joined multiple study periods, and each participant donated 3-20 samples. Some samples were qualified for both the twoway factorial and overnight designs. In addition, nine participants had $>4$ samples collected within 2 weeks, allowing us to study trends in their metabolic profiles.

\section{Exposure assessment}

On welding days, apprentices were lessoned in manual metal arc welding on mild and stainless steel. The whole session lasted about 6 hours from morning to afternoon, with a meal break taken in the classroom area. In the control period, participants stayed in the classroom area and completed questionnaires and testing. During the shift, each participant (in both welding and classroom areas) wore a personal DustTrak Aerosol Monitor (TSI, St. Paul, Minnesota, USA) to analyse PM2.5 concentration from the breathing zone. Details of this exposure assessment method were described previously. ${ }^{13}$ Briefly, the monitor provides continuous $1 \mathrm{~min}$ averages of particulate levels using an inlet impactor to select particles of $<2.5 \mu \mathrm{m}$ and light scattering to determine concentrations. All participants wore personal protective equipment, including face shields and gloves. Local exhaust ventilation was placed at the operating bench near the welding source, in addition to ceiling general ventilation.

\section{Blood collection method}

A certified phlebotomist drew blood samples into EDTA-coated vacutainer tubes (BD Scientific, Franklin Lakes, New Jersey, USA) at the training centre. After allowing blood samples to clot at room temperature for $20-30 \mathrm{~min}$, the clot was removed by centrifuging at 1200 revolutions per minute for $12 \mathrm{~min}$. Plasma was aliquoted to cryogenic tubes and stored at $-80^{\circ} \mathrm{C}$. All

\begin{tabular}{|c|c|c|c|c|c|c|c|c|c|}
\hline \multirow[b]{2}{*}{ Sampling periodt } & \multirow[b]{2}{*}{ Design } & \multirow[b]{2}{*}{$\mathrm{N}$} & \multicolumn{2}{|c|}{ Non-welding day } & \multicolumn{2}{|l|}{ Welding day } & \multicolumn{3}{|c|}{ Non-welding days‡ } \\
\hline & & & $\begin{array}{l}\text { The previous } \\
\text { morning }\end{array}$ & $\begin{array}{l}\text { The previous } \\
\text { afternoon }\end{array}$ & Morning (preshift) & $\begin{array}{l}\text { Afternoon } \\
\text { (postshift) }\end{array}$ & $\begin{array}{l}\text { The next } \\
\text { morning }\end{array}$ & Morning & Afternoon \\
\hline 2006 & Overnight & 35 & & & 35 samples & 35 samples & 35 samples & & \\
\hline $\begin{array}{l}\text { January 2010-February } \\
2010\end{array}$ & Overnight & 30 & & 30 samples & 30 samples & 30 samples & & & \\
\hline June 2010-July 2010 & Overnight & 33 & & 33 samples & 42 samples & 42 samples & 9 samples & & \\
\hline $\begin{array}{l}\text { January 2011-February } \\
2011\end{array}$ & Two-way & 6 & 6 samples & 6 samples & 14 samples & 14 samples & & 8 samples & 8 samples \\
\hline June 2011 & Two-way & 14 & 14 samples & 14 samples & 14 samples & 14 samples & & & \\
\hline June 2012 & Two-way & 16 & 16 samples & 16 samples & 16 samples & 16 samples & & & \\
\hline
\end{tabular}

44 sets of samples were qualified for postexposure overnight design; 99 sets of samples were qualified for pre-exposure overnight design; 44 sets of samples were qualified for two-way factorial design.

†74 welders participated in the study. Some welders participated in multiple sampling periods. We collected 509 samples in total.

$\ddagger$ Non-welding days is within \pm 7 days' interval from the welding day; besides, a few participants had additional samples collected at baseline (12 samples). 
samples were kept frozen before shipping on dry ice to Metabolon (Morrisville, North Carolina, USA) for analysis.

\section{Metabolite profiling}

A detailed description of the metabolite profiling method was previously published. ${ }^{14}$ Briefly, proteins were precipitated from plasma and stored overnight in liquid nitrogen before analysis. At the time of analysis, each extract was divided into multiple fractions for different analytical techniques, including reversephase ultrahigh-performance liquid chromatography-tandem mass spectroscopy methods with both positive and negative ion mode electrospray ionisation (ESI) and hydrophilic interaction ultraperformance liquid chromatography-mass spectroscopy with negative ion mode ESI. Different methods quantifies different groups of metabolites.

In summary, 655 biochemical metabolites were identified from each sample, including amino acids, carbohydrates, cofactor/vitamins, lipids, nucleotides, all characterised molecules, peptides and xenobiotic metabolites.

\section{Instrumental quality control and peak reading}

Before the first step of the analysis, internal standards were added to each sample for quality control purposes. Instrument variability, determined as the median relative SD (RSD) for internal standards of each sample, was $4 \%$.

A reference sample was created by pooling small volumes of different well-characterised human plasma samples, and it served as a technical replicate in the analysis. Overall process variability was $8 \%$, determined as median RSD for all endogenous metabolites present in the matrix sample.

Peak data were read by the Metabolon LIMS system. Compounds were quantified using area under the curve. As the procedure spanned multiple days, the instrument's interday variation was normalised per batch per compound. Missing values due to low concentration were imputed as the minimum observed value for each compound. Each compound's concentration was median scaled to 1 . Data were log-transformed and mean-centred before statistical analysis.

\section{Covariates}

We collected information regarding age, body mass index, gender, race, physician diagnosis of asthma or diabetes, and smoking status. As this is a repeated measurement study with each participant serving as his own control, we did not include these covariates in statistical models. However, some analysis was stratified by smoking status.

\section{Statistical analysis}

There were three phases of statistical analysis: (1) screening for metabolites differentially expressed based on exposure, using two-way factorial ANOVA with Benjamini-Hochberg false discovery rate (FDR) adjustment ${ }^{15}$; (2) investigating the profile of selected metabolites based on exposure, including overnight expression pattern; and (3) assessing the expression pattern of selected metabolites in a longer 2-week window.

In phase I, we first performed principal component analysis to identify factors influencing data clustering.

Once we confirmed that batch effect of different instrumental runs had minimal effect on data clustering, we performed two-factor ANOVA analysis about each metabolite based on day (welding/classroom area), time (morning/afternoon) and an interaction term, and adjusted the $\mathrm{p}$ value with the FDR method. The aforementioned analysis was performed online with MetaboAnalyst V.3.0. ${ }^{16}$

In phase II, we selected the only two metabolites (sphingosine 1-phosphate (S1P) and sphinganine 1-phosphate) with significant day/time interaction effects from the previous analysis. Another metabolite (sphingosine) was selected as among the top 10 hits and in the same metabolic pathway (ie, a precursor of S1P). With each of the three metabolites as the outcome variable, we evaluated the within-subject effect of welding fume exposure.

In the two-factorial design, we plotted metabolites at two time points on the day of welding or in the classroom area. We used the within-subject test to indicate the effect of day, time and daytime interaction.

In two-way designs, we plotted the average level of metabolites stratified by current smokers and non-smokers. We also performed stratified analysis based on participants' weight, and overweight has been defined as BMI of no less than $25 \mathrm{~kg} / \mathrm{m}^{2}$. The aforementioned analysis was based on balanced data subsets.

In phase III, we evaluated the profile of the three metabolites in a cross-over design. We stratified the 44 participants into two groups. The first group performed welding before the classroom day $(\mathrm{n}=8)$, while the second group performed welding after the classroom day $(n=36)$. We evaluated profiles of the three metabolites and compared trends in the two groups. In addition, among the nine participants who had $>4$ samples collected within half a month, 6 and 3 had welding days before or after, respectively, their control day, and the trajectory of relative levels of the three metabolites over time is presented in the online supplementary materials.

The above analysis was performed with SAS 9.4 software V.9.4.

\section{RESULTS}

The study population consisted of 74 men, with an average age of 42 years. The majority (86\%) was Caucasian. Average BMI was $29.4 \mathrm{~kg} / \mathrm{m} 2$, close to the definition of obese $(30 \mathrm{~kg} / \mathrm{m} 2)$. Demographic characteristics and exposure levels are shown in table 2. Average PM 2.5 level of participants' daily means during the welding shift was $421 \mu \mathrm{g} / \mathrm{m}^{3}$, which was significantly higher than the $120 \mu \mathrm{g} / \mathrm{m}^{3}$ exposure in the classroom area (t-test $\mathrm{p}<0.01)$. More data about seasonal PM2.5 exposure level are shown in online supplemental table 1. The PM2.5 exposure level in winter was higher than it in summer.

Color-coded PCA plots are shown in the Supplementary Material (online supplemental figure 1 and 2), indicating the sample batch does not relate to data clustering. However, there were $37 \%$ current smokers in the study population, and smoking status was related to data clustering within the overall sample.

Using within-subject ANOVA, we found that S1P and sphinganine 1-phosphate (SA1P) had significant interaction effects between the type of day (welding/classroom area) and time (morning/afternoon). FDR-adjusted $p$-values for interaction for S1P and SA1P were 0.03 and $<0.01$, respectively (online supplemental table 2). This indicates that these two metabolites were expressed differently based on exposure, controlled for circadian rhythm. Sphingosine was among the top 10 hits but without a significant interaction term. It was selected for further consideration because it is the precursor of S1P (online supplemental figure 3).

In phase II, we further evaluated the profile of S1P, SA1P, and sphingosine. Relative levels of these metabolites in the two-factor factorial design are shown in table 3 and visualised in figure 1. Data from four samples each from 44 sampling 


\begin{tabular}{lcc}
\hline \multicolumn{2}{l}{ Table 2} & \multicolumn{2}{c}{ Characteristics of participants } & \\
\hline Characteristics & Mean & SD \\
\hline Age (years) & 41.7 & 13.3 \\
\hline BMI $\left(\mathrm{kg} / \mathrm{m}^{2}\right)$ & 29.4 & 6.6 \\
\hline PM2.5_welding $\left(\mu \mathrm{g} / \mathrm{m}^{3}\right)$ & 421 & 360 \\
\hline PM2.5_classroom area $\left(\mu \mathrm{g} / \mathrm{m}^{3}\right)$ & 120 & 60 \\
\hline & N & $\%$ \\
\hline Smoking status & & \\
\hline Non-current-smoker & 40 & 63 \\
\hline Current smoker & 34 & 37 \\
\hline Gender & & \\
\hline Male & 74 & 100 \\
\hline Race & & 85 \\
\hline Caucasian & 63 & 9 \\
\hline African-American & 7 & 3 \\
\hline Asian & 2 & 3 \\
\hline Other & 2 & 94 \\
\hline Diabetes (ever) & & 6 \\
\hline No & 69 & 94 \\
\hline Yes & 5 & 9 \\
\hline Asthma (ever) & 59 & \\
\hline No & 5 & \\
\hline Yes & & \\
\hline PM2.5 is the average of & & \\
\hline
\end{tabular}

PM2.5 is the average of 1 min averages over the 6 -hour shift.

$\mathrm{BMI}$, body mass index; PM, particulate matter

groups (176 samples in total) showed that S1P, SA1P, and sphingosine started with high levels in the morning of the classroom day and dropped in the afternoon. However, on the welding day, the three metabolites started at a lower level, then S1P did not change; SA1P increased, while sphingosine decreased over the welding shift. Unadjusted $p$-values for the interaction were $<0.01,<0.01$, and 0.06 , respectively.

From repeated measurements ANOVA, we found that S1P and SA1P levels did not change from the previous afternoon to the morning before welding, while sphingosine decreased during this period. S1P slightly decreased on the welding day, while no significant change was found for SA1P and sphingosine during the same period. There was no obvious pattern found in stratified analysis by current smoking status and overweight status, respectively (online supplemental figures 4 and 5).

In the overnight design to the morning after welding: all metabolites decreased during the welding shift and slightly increased until the next morning (table 3 and figure 2). We did not find that smoking status had an interaction effect with time.

In phase III, we confirmed that the trends of the three metabolites in the two-factor factorial design were the same whether the welding day was measured before or after the classroom day (figure 2). The beginning levels of S1P, SA1P, and sphingosine were always higher on the classroom day compared with the welding day.

We performed analysis of participants who provided five or six samples within about 2 weeks. Despite the small sample size, we observed that trajectories of the three metabolites were still highly correlated. Each compound tended to peak in the morning of the classroom day (online supplemental figure 6). In addition, afternoon levels of these compounds in the classroom were stable over time.

\section{DISCUSSION}

This study evaluated plasma metabolites that were differently expressed under welding fume exposure. As an occupational hazard of welding, the PM2.5 level in both the welding area and classroom area exceeded the WHO recommendation of $10 \mu \mathrm{g} /$ $\mathrm{m}^{3}$ for an annual mean or $25 \mu \mathrm{g} / \mathrm{m}^{3}$ for a 24 -hour mean. ${ }^{17}$

We found that S1P and SA1P levels were disrupted on the day of welding. We focused on these two compounds as well as sphingosine, which is the precursor of S1P, in our analysis and identified expression patterns related to different exposure conditions.

Table 3 Within-subject effect of day (welding/classroom area) and time (morning/afternoon) on three metabolites, as well as overnight effects

\begin{tabular}{|c|c|c|c|c|c|c|}
\hline & Sphingosine & & Sphinganine & & Sphingosine & \\
\hline & Mean* (SD) & & Mean (SD) & & Mean (SD) & \\
\hline Two-factor fac & & $\mathrm{Pr}>\mathrm{F}$ & & $\mathrm{Pr}>\mathrm{F}$ & & $\mathrm{Pr}>\mathrm{F}$ \\
\hline N/AM & $0.24(0.33)$ & & $0.16(0.44)$ & & $0.25(0.63)$ & \\
\hline N/PM & $-0.04(0.33)$ & & $-0.15(0.49)$ & & $-0.13(0.49)$ & \\
\hline W/AM & $-0.16(0.34)$ & $P_{\text {interaction }}<0.01$ & $-0.27(0.43)$ & $\mathrm{P}_{\text {interaction }}<0.01$ & $-0.18(0.69)$ & $P_{\text {interaction }}=0.06$ \\
\hline W/PM & $-0.16(0.26)$ & & $-0.05(0.52)$ & & $-0.29(0.65)$ & \\
\hline Previous afterr & velding day & $\operatorname{Pr}>|t|$ & & & & \\
\hline Previous PM & $0.02(0.36) \dagger$ & $<0.31$ & $-0.04(0.49)$ & 0.72 & $0.07(0.78)$ & 0.01 \\
\hline W/AM & $-0.13(0.28)$ & $<0.01$ & $-0.03(0.41)$ & 0.24 & $-0.18(0.86)$ & 0.91 \\
\hline W/PM & $-0.02(0.29)$ & & $-0.09(0.41)$ & & $-0.17(0.58)$ & \\
\hline Welding day a & rning & & & & & \\
\hline W/AM & $0.06(0.44)$ & $<0.01$ & $0.00(0.61)$ & $<0.01$ & $-0.04(0.46)$ & $<0.01$ \\
\hline W/PM & $-0.27(0.28)$ & 0.39 & $-0.24(0.42)$ & 0.01 & $-0.29(0.38)$ & 0.42 \\
\hline Next morning & $-0.17(0.29)$ & & $-0.19(0.48)$ & & $-0.17(0.40)$ & \\
\hline
\end{tabular}

The sample sizes for two-factor factorial ANOVA, pre-exposure overnight design and postexposure overnight design were 176, 297 and 132, respectively.

$\mathrm{Pr}>\mathrm{F}$ indicates probability that the predictor has no effect on the outcome variable.

$\operatorname{Pr}>|t|$ indicates probability that the level was different compared with the last measurement.

Interaction indicates the effect between the previous two factors.

*The mean of metabolites was calculated from concentrations after normalisation, log transformation and mean centring.

tThese values are for both current smokers and non-current smokers.

$\mathrm{AM} / \mathrm{PM}$, afternoon compared with morning; ANOVA, analysis of variance; interaction, interaction effect between the previous two factors; W/N, welding day compared with nonwelding day. 

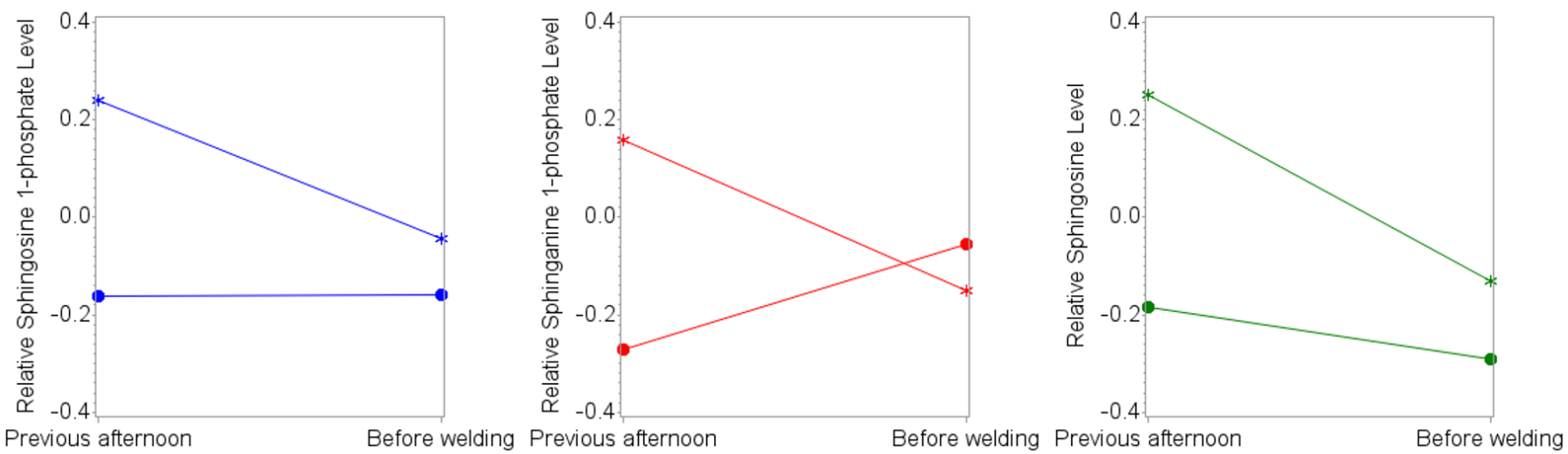

Weld_day $0 \cdots 1$

Weld_day $0 \cdots 1$

Weld_day $0 \cdots 1$

Figure 1 Profiles of sphingosine 1-phosphate, sphinganine 1-phosphate and sphingosine over time. The three plots show levels of the indicated compounds by day (welding/classroom area) and time (morning/afternoon) based on 44 sets of two-way balanced data.

We observed that, on the classroom day, the three compounds started at a relatively high level and declined during the day. This observation concurs with a previous study about the circadian rhythm of S1P that it increases overnight and drops during the day within a normal range of $6-9 \mathrm{nmol} / \mathrm{mL} .{ }^{18}$ This could represent the shared regular circadian rhythms of S1P, SA1P and sphingosine, and we can assume that the three metabolites increase overnight and drop during the day. In comparison, on the welding day, the starting point was much lower than that of the classroom day for the three metabolites, and their levels did not change dramatically over time. Instead, the level remained the same or slightly increased or decreased during the welding shift. To date, no other study reported factors that may influence the circadian rhythm of S1P.

In the postexposure overnight design, we did observe a decrease of all metabolites from the beginning to the end of a welding shift; however, the level of three metabolites ranged -0.1 to 0.1 in the morning, still lower than their profiles on the morning of classroom days, ranged $0.1-0.3$.

An increase in the compounds from the previous afternoon to the sampling time before the welding shift was not observed. Instead, we observed an increase between the end of the welding shift and the next morning, and this pattern was consistent whether the welding day occurred before or after the classroom day. Also, the profiles of S1P, SA1P and sphingosine were highly correlated with each other. When stratified by smoking, the pattern did not change; however, non-smokers may have be exposed to secondhand smoke in the classroom area. Thus, we cannot conclude the impact of smoking status.

$\mathrm{S} 1 \mathrm{P}$ is a signalling sphingolipid belonging to the sphingosine subpathway of the lipid superpathway. It is a bloodborne lipid mediator particularly associated with lipoproteins, including high-density lipoprotein. Sphingolipids are a lipid family characterised by a particular aliphatic aminoalcohol, namely, sphingosine. S1P is the extracellular ligand for G protein-coupled receptor S1PR1, and it regulates the vascular system by affecting angiogenesis, vascular stability and permeability. It also functions in immune systems that regulate trafficking of T cells and B cells. $\mathrm{S} 1 \mathrm{P}$ has been used as a drug to protect cells from chemotherapeutic and radiation therapies, ${ }^{19}$ and it protects against ischaemia-reperfusion injury. ${ }^{20}$

The disruption of S1P and related sphingolipids observed in this study may contribute to the inflammatory response and cardiovascular effect of welding fumes. Studies show that high levels of welding fume exposure are related to acute changes in inflammatory biomarkers in blood circulation, ${ }^{21}$ as well as acute changes in vascular functions. ${ }^{722}$ While the roles of the S1P-S1P receptor signalling system in immunological trafficking and maintenance of vascular integrity is established, ${ }^{23}$ precisely how it contributes to regulation of inflammatory processes and pathology is complex. One study has shown that sphingosine kinase 1 regulates inflammation and contributes to acute lung injury in pneumococcal pneumonia via the S1P receptor $2 .{ }^{24}$ This reflects a potential role for metabolites of the S1P pathway and inflammatory pulmonary disease, to which welders are especially susceptible. This is important as it is known that recurrent exposure to welding fumes may cause insufficient recovery from inflammation. ${ }^{25}$ The actions related to S1P are complex, and more information about its roles in diseases can be found in a review by Maceyka et al. ${ }^{26}$

SA1P is an intermediate in the metabolism of glycosphingolipids and sphingolipids. Sphinganine, the precursor of SA1P, is also part of the metabolic pathway for synthesis of sphingosine and S1P. A recent study shows that SA1P may protect the kidney and liver from hepatic ischaemia and reperfusion in mice by selectively activating the S1PR1 receptor. ${ }^{27}$

To our knowledge, this is the first study that investigates the metabolic profile of workers under welding fume exposure that is properly controlled and validated. The balanced repeatedmeasurement study design was efficient and minimised biological variability. The three-phase analysis provides valuable insight into how metabolites change over time. The cross-over design has this same advantage and additionally required fewer participants, thus reducing background noise.

We acknowledge limitations of the study. There might have been healthy worker bias. In occupational epidemiology studies, participants are often those less sensitive to the exposure, or selected by the job, who tend to have less relative mortality and morbidity rates. This phenomenon would bias towards the null, thus underestimating any exposure effect. The same exposure is possibly related to an increased risk of disease for a wider population. In addition, there may have been fugitive exposure in the classroom area released from welding area, but this was the only feasible way to collect control samples. In this instance, our results would be biassed towards the null, not finding an effect. Further, we only assessed relative level of metabolites, missing the absolute range of metabolites for comparison to normal range guidelines. Besides, the sample size is limited to 

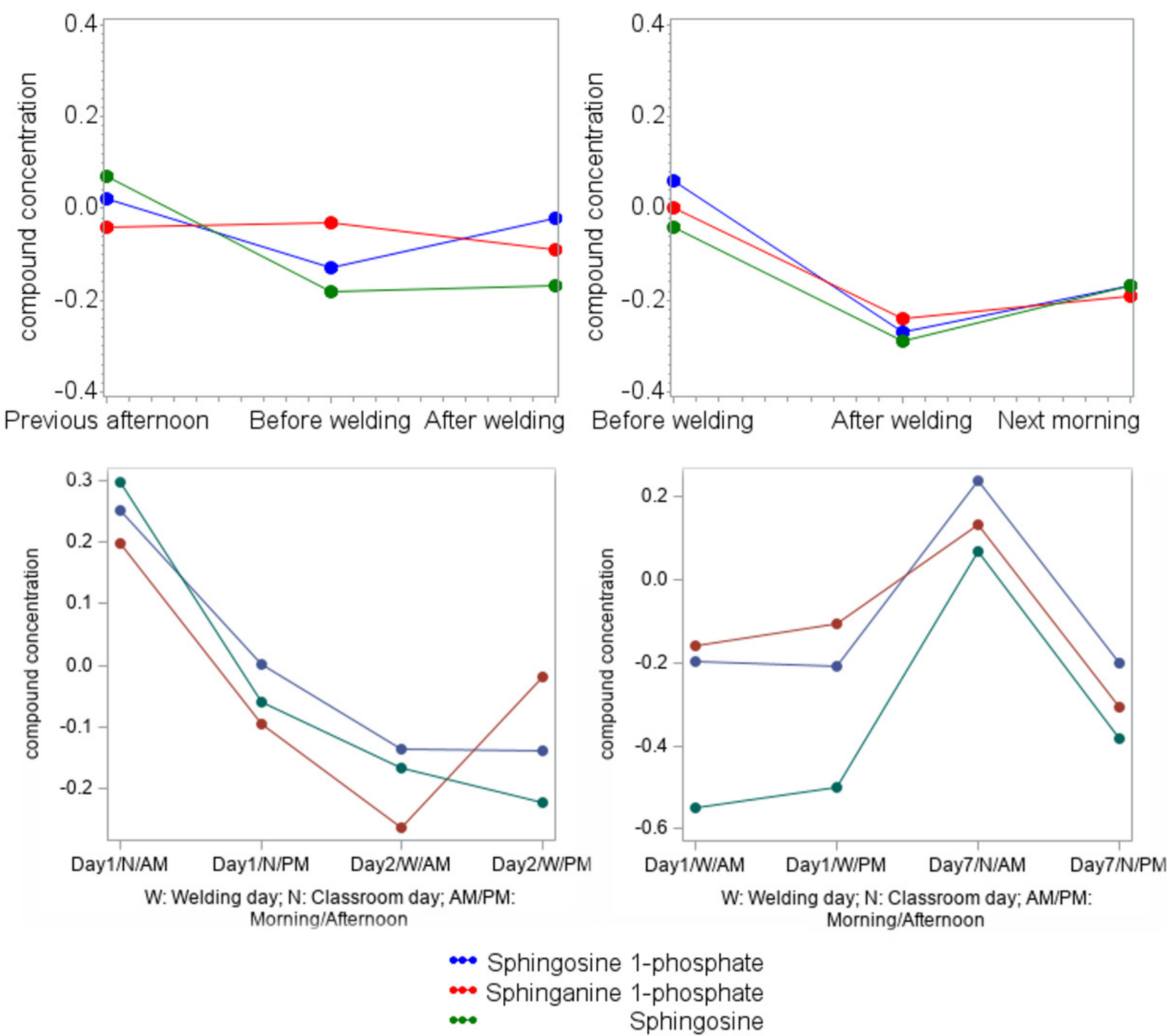

Figure 2 Profile of sphingosine 1-phosphate, sphinganine 1-phosphate and sphingosine over time in overnight settings and a cross-over setting. The upper left plots show levels of the indicated compounds on the previous afternoon and the morning and afternoon of the welding day (before and after a welding shift), based on 99 groups of balanced data. There were 60 non-smokers and 38 smokers in this sample. The upper right plot shows levels of indicated compounds on the morning and afternoon of the welding day (before and after a welding shift) and the next morning, based on 44 groups of balanced data. There were 21 non-smokers and seven smokers in this sample. In the lower left plot, the welding day was after the classroom day; in the lower right plot, the welding day was before the classroom day.

investigate the metabolite profile in a longer period by five or more measurements. Future studies with a larger sample size can validate the pattern we found and evaluate whether the effect of welding fume exposure is reversible.

With regard to controlling the exposure, we found that the average PM2.5 level in classroom area was higher than WHO recommendations. Although the welding training centre set negative pressure from the classroom to the welding area, welders may open the door and introduce fumes from the welding area into the classroom during lunch break. Comparing PM2.5 exposure levels between summer and winter, we found that PM2.5 exposure level was higher in winter. However, it would not bias our study because in this self-controlled study, the control was measured within a few days before or after the exposure.
In conclusion, metabolism of the S1P pathway was disrupted on the day of welding. This change may be reversible. Since $\mathrm{S} 1 \mathrm{P}$ is a vital signalling molecule regulating many biological processes, the underlying mechanism and influence of this alteration require further investigation.

Acknowledgements The authors acknowledge the cooperation of study participants and the support of the International Brotherhood of Boilermakers Union, Local 29 in Quincy, Massachusetts. We thank Tianteng Fan, Jinming Zhang, Jason Wong, Jennifer M Cavallari, Ema Rodrigues and other former team members for building this cohort together and collecting data and samples.

Contributors SG contributed to study design, sample processing, statistical analysis and manuscript writing. ZZ and JH contributed to data quality control and statistical analysis. LS contributed to participant recruitment, participant follow-up, 
sample collection and sample management. DCC is the principal investigator of the study.

Funding This study was supported by a grant (T42OH008416) issued by National Institute for Occupational Safety and Health, Centers for Disease Control and Prevention, US Department of Health and Human Services.

Competing interests None declared.

Patient consent for publication Not required.

Ethics approval Signed informed consent was obtained from all study participants in each study period. All study procedures were approved by the institutional review board of the Harvard T H Chan School of Public Health.

Provenance and peer review Not commissioned; externally peer reviewed.

Data availability statement No data are available. Data of this study are not available in a public, open access repository.

Supplemental material This content has been supplied by the author(s). It has not been vetted by BMJ Publishing Group Limited (BMJ) and may not have been peer-reviewed. Any opinions or recommendations discussed are solely those of the author(s) and are not endorsed by BMJ. BMJ disclaims all liability and responsibility arising from any reliance placed on the content. Where the content includes any translated material, BMJ does not warrant the accuracy and reliability of the translations (including but not limited to local regulations, clinical guidelines, terminology, drug names and drug dosages), and is not responsible for any error and/or omissions arising from translation and adaptation or otherwise.

Open access This is an open access article distributed in accordance with the Creative Commons Attribution Non Commercial (CC BY-NC 4.0) license, which permits others to distribute, remix, adapt, build upon this work non-commercially, and license their derivative works on different terms, provided the original work is properly cited, appropriate credit is given, any changes made indicated, and the use is non-commercial. See: http://creativecommons.org/licenses/by-nc/4.0/.

\section{ORCID iDs}

Shangzhi Gao http://orcid.org/0000-0003-2832-3058

David C Christiani http://orcid.org/0000-0002-0301-0242

\section{REFERENCES}

1 Guha N, Loomis D, Guyton KZ, et al. Carcinogenicity of welding, molybdenum trioxide, and indium tin oxide. Lancet Oncol 2017;18:581-2.

2 Beckett WS. Occupational respiratory diseases. N Engl J Med 2000;342:406-13.

3 Shen S, Zhang R, Zhang J, et al. Welding fume exposure is associated with inflammation: a global metabolomics profiling study. Environ Health 2018;17:68.

4 Wong JYY, De Vivo I, Lin X, et al. The relationship between inflammatory biomarkers and telomere length in an occupational prospective cohort study. PLoS One 2014;9:e87348.

5 Baur X. Inflammatory response to acute exposure to welding fumes during the working day. J Occup Med Toxicol 2013;8:15.

6 Nuernberg AM, Boyce PD, Cavallari JM, et al. Urinary 8-isoprostane and 8-OHdG concentrations in boilermakers with welding exposure. J Occup Environ Med 2008:50:182-9.

7 Cavallari JM, Eisen EA, Chen J-C, et al. Night heart rate variability and particulate exposures among boilermaker construction workers. Environ Health Perspect 2007;115:1046-51.
8 Cavallari JM, Eisen EA, Fang SC, et al. Pm2.5 metal exposures and nocturnal heart rate variability: a panel study of boilermaker construction workers. Environ Health 2008;7:36.

9 Cavallari JM, Fang SC, Eisen EA, et al. Time course of heart rate variability decline following particulate matter exposures in an occupational cohort. Inhal Toxicol 2008;20:415-22.

10 Barkhordari A, Zare Sakhvidi MJ, Zare Sakhvidi F, et al. Oxidative stress, telomere shortening, and DNA methylation in relation to low-to-moderate occupational exposure to welding fumes. Iran J Public Health 2014;43:666-73.

11 Kile ML, Fang S, Baccarelli AA, et al. A panel study of occupational exposure to fine particulate matter and changes in DNA methylation over a single workday and years worked in boilermaker welders. Environ Health 2013;12:47.

12 Wong JYY, De Vivo I, Lin X, et al. The association between global DNA methylation and telomere length in a longitudinal study of boilermakers. Genet Epidemiol 2014:38:254-64.

13 Fan T, Fang SC, Cavallari JM, et al. Heart rate variability and DNA methylation levels are altered after short-term metal fume exposure among occupational welders: a repeated-measures panel study. BMC Public Health 2014;14:1279.

14 Shen S, Zhang R, Zhang J, et al. Welding fume exposure is associated with inflammation: a global metabolomics profiling study. Environ Health

15 Benjamini Y, Hochberg Y, YJJotRsssB H. Controlling the false discovery rate: a practical and powerful approach to multiple testing. J R Stat Soc Series B 1995;57:289-300.

16 Xia J, Sinelnikov IV, Han B, et al. MetaboAnalyst 3.0--making metabolomics more meaningful. Nucleic Acids Res 2015:43:W251-7.

17 Organization WH. WHO air quality guidelines for particulate matter, ozone, nitrogen dioxide and sulfur dioxide: global update 2005: summary of risk assessment. Geneva: World Health Organization, 2006.

18 Budkowska M, Ostrycharz E, Wojtowicz A, et al. A circadian rhythm in both complement cascade (comC) activation and sphingosine-1-phosphate (S1P) levels in human peripheral blood supports a role for the ComC-S1P axis in circadian changes in the number of stem cells circulating in peripheral blood. Stem Cell Rev Rep 2018:14:677-85.

19 Morita Y, Perez Gl, Paris F, et al. Oocyte apoptosis is suppressed by disruption of the acid sphingomyelinase gene or by sphingosine-1-phosphate therapy. Nat Med 2000;6:1109-14

20 Theilmeier G, Schmidt C, Herrmann J, et al. High-Density lipoproteins and their constituent, sphingosine-1-phosphate, directly protect the heart against ischemia/ reperfusion injury in vivo via the S1P3 lysophospholipid receptor. Circulation 2006:114:1403-9.

21 Kim JY, Chen J-C, Boyce PD, et al. Exposure to welding fumes is associated with acute systemic inflammatory responses. Occup Environ Med 2005;62:157-63.

22 Fang SC, Eisen EA, Cavallari JM, et al. Acute changes in vascular function among welders exposed to metal-rich particulate matter. Epidemiology 2008;19:217-25.

23 Obinata H, Hla T. Sphingosine 1-phosphate and inflammation. Int Immunol 2019;31:617-25.

24 Gutbier B, Schönrock SM, Ehrler C, et al. Sphingosine kinase 1 regulates inflammation and contributes to acute lung injury in pneumococcal pneumonia via the sphingosine1-phosphate receptor 2. Crit Care Med 2018:46:e258-67.

25 Yang MJ, Yang YS, Sung JH, et al. Recurrent exposure to welding fumes induces insufficient recovery from inflammation. Inhal Toxicol 2009;21:337-46.

26 Maceyka M, Harikumar KB, Milstien S, et al. Sphingosine-1-Phosphate signaling and its role in disease. Trends Cell Biol 2012;22:50-60.

27 Park SW, Kim M, Chen SWC, et al. Sphinganine-1-Phosphate protects kidney and liver after hepatic ischemia and reperfusion in mice through S1P1 receptor activation. Lab Invest 2010:90:1209-24. 